\title{
No Clear Winner in Differing Imaging Modalities for Cervical Radiculopathy
}

\author{
Joseph Fortin, DO*, Dan W. Riethmiller, BS** and Joel A. Vilensky PhD\#
}

We review the literature pertaining to the strengths and limitation of myelography, computerized tomography (CT) and magnetic resonance imaging (MRI) relative to developing a diagnostic algorithm in the evaluation of cervical radiculopathy. To obtain the relevant literature, a Medline search was conducted using selected keywords and phrases. In addition, the bibliography of all retrieved articles was searched and pertinent articles were obtained and evaluated. The analysis revealed that developing a research based algorithm of imaging studies for the evaluation of cervical radiculopathy is not currently realistic. Furthermore, we found that most studies on the predictive value of imaging studies for cervical radiculopathy are outdated by more recent technology.

Keywords: Computerized tomography, magnetic resonance imaging, myelography
In light of the rising importance of cost containment, it has become crucial to utilize the most elucidating diagnostic test or tests for the resolution of clinical ambiguities including those associated with the diagnosis of cervical radiculopathy (a disease of the cervical spinal nerve root (1) that is most commonly caused by a cervical disc herniation or other space occupying lesion that may result in nerve root pathology (2)). This report examines those studies that evaluated the three most prevalent diagnostic imaging modalities utilized for the evaluation of cervical radiculopathy: myelography, computerized tomography (CT) and magnetic resonance imaging (MRI). The overall goal was to determine the relative value of each imaging studies in aiding in the development of a diagnostic algorithm for the evaluation of cervical radiculopathy.

From Spine Technology and Rehabilitation, PC, Fort Wayne, Indiana and Indiana University School of Medicine, Indianapolis, Gary, and Fort Wayne, Indiana. *Dr. Fortin is medical director of Spine Technology and Rehabilitation, PC and clinical professor of Indiana University School of Medicine, **Mr. Riethmiller is a medical student at the Indiana University School of Medicine, Gary, Indiana, and "Dr. Vilensky is a professor of anatomy in the Department of Anatomy at Indiana University School of Medicine, Fort Wayne, Indiana. Address correspondence: Dr. Joseph D. Fortin, 7230 Engle Road, Suite 210, Fort Wayne, IN 46804. E-mail: fortin@pol.net

\section{METHODS}

Relevant literature pertaining to the predictive value of each of the three imaging modalities in the assessment of cervical radiculopathy was evaluated. To obtain these reports, Medline was searched using pertinent keywords and phrases (e.g., myelography, computerized tomography (CT), magnetic resonance imaging (MRI) and cervical radiculopathy). Approximately 50 articles were eventually obtained based on this search and a review of the bibliographies in some of the retrieved articles.

\section{RESULTS}

\section{Myelography}

Conventional myelography is as effective as orthogonal imaging in assessing the level of space occupying lesions effacing the dural sac, nerve root, and spinal cord (3). Conversely, this modality is unable to depict lesions that do not efface the thecal sac or dural root sleeves. However, myelography poorly distinguishes the nature of a defect (e.g., extradural osteophyte versus cervical disc prolapse). Whereas myelography has an additive benefit when combined with CT (4), its applicability as a stand-alone procedure is limited to screening for intrathecal tumors or other space occupying lesions.

\section{Computerized Tomography}

CT is particularly powerful in discerning a degenerative osseous ridge from soft disc material or a combination of these events (5). However, CT has a limited ability to detect soft tissue lesions (3, 6-9), particularly in defining 
intrathecal pathology and it is sometimes inadequate for evaluating the cervicothoracic motion segments (secondary to beam attenuation from the overlying shoulders).

\section{CT/myelography}

The combination of CT with myelography is equal to MRI in demonstrating cervical radiculopathy caused by lesions that correlate to surgical findings $(6,10)$. Both Brown et al (6) and Modic et al (10) concluded that CT/myelography was valuable in the surgical decision making process for cervical radiculopathy patients. Van de Kelft et al (11) reported that CT/myelography demonstrated foraminal disc herniation better than MRI. Similarly, Shafaie et al (3) concluded probably CT/myelography is preferred to MRI in the pre-operation evaluation of cervical spondylosis and disc herniation based on superior differentiation of bone and soft tissues (including uncarthrosis). However, Shafaie and co-workers also noted neither CT/myelography nor MRI should be solely relied on in the evaluation of cervical degenerative disc disease. It is also important to note that CT/myelography subjects the patient to ionizing radiation and invasion of the thecal sac; thereby, potentially leading to unnecessary complications.

\section{Magnetic Resonance Imaging}

MRI is generally more sensitive for depicting intrathecal pathology and soft tissue changes than the other modalities, either separately or in combination $(8,10,12)$. MRI often has the capacity to differentiate which soft tissue structures are causing stenosis, e.g., facet joint effusion, capsular hypertrophy, disc material, perineural hematoma, abscess or neoplasm. Shortcomings of MRI in the cervical spine include: 1) relatively thicker sections and intersection gaps compared to CT (except with newer 3-D techniques); 2) variable signal intensity in degenerative osseous ridges that may result in poor resolution; and, 3) magnetic susceptibility, i.e., over-estimation of the degree of foraminal narrowing and lateral neural encroachment. However, recent studies $(13,14)$ have shown the value of magnetization transfer with 3-D gradient echo techniques for diminishing this effect. MRI depicts changes in the biochemical matrix of the disc better than CT, which may be relevant in cases of chemical radiculitis, but osseous lesions may be missed (15).

\section{GENERAL FINDINGS}

1. Most reports on the predictive value of imaging studies for cervical radiculopathy are outdated by more recent technology $(6,14,16)$.

2. The definitions of cervical radiculopathy sometimes varied, although most defined cervical radiculopathy by imaging studies alone (i.e., there was no electrodiagnostic correlation).

3. The study designs were open, often retrospective and inter-rater reliability was not assessed.

4. The surgical approach, aggressiveness and experience may vary as will the visualization and interpretation of the surgical findings.

5. Few surgeons operate on patients with negative imaging studies. Consequently false negative and true negative findings cannot be properly evaluated.

6. Correlating imaging results with surgical pathology may miss false positive findings because if the lesion is not creating symptoms, the surgeon will not be "looking” for it $(4,16-18)$.

7. The imaging equipment and techniques significantly varied from study to study.

8. Clinical localization of the lesion and clinical response to surgery can be influenced by factors other than imaging interpretation accuracy. For example, physiological (versus pure structural) studies such as electrodiagnostics and/or discography may identify nerve root or disc abnormalities at levels that are not always commensurate with imaging studies.

9. There is a known high incidence of false positive findings in cervical imaging studies (16-18).

\section{CONCLUSION}

Our evaluation suggests that the clinical presentation of the patient (history and physical examination) must be the main factor that determines imaging choices. Clearly, no one modality is best for all situations. Often it is reasonable to begin an imaging evaluation with a non-invasive cervical MRI, using CT/myelography to provide additional, valuable information in difficult or ambiguous cases and in elderly patients with primary osseous stenosis. The literature suggests that no clear diagnostic algorithm can be rigidly established or substantiated. Further, a decision cannot also easily be made based on cost, because the costs of CT/myelography and MRI are similar. 


\section{REFERENCES}

1. Spraycar M, Randolph E. Stedman's Illustrated Medical Dictionary, Williams and Wilkins Company, Baltimore, 1995.

2. Radhakrishnan K, Litchy WJ, O’Fallon $\mathrm{M}$ et al. Epidemiology of cervical radiculopathy. A population-based study from Rochester, Minnesota, 1976 through 1990. Brain 1994; 117: 325-335.

3. Shafaie FF, Wippold II FJ, Gado M et al. Comparison of computed tomography, myelography, and magnetic resonance imaging in the evaluation of cervical spondylotic myelopathy and radiculopathy. Spine 1999; 24:1781-1785.

4. Wilmink JT: Clinical relevance of cervical disk herniation diagnosed on the basis of MR imaging (letter). Am J Neuroradiol 1989; 10:1278-1279.

5. Penning L, Wilmink JT, vanWoerden HH et al. Computed tomography myelographic findings in degenerative disorders of the cervical spine: Clinical significance. Am J Roentgenol 1986; 146:793-801.

6. Brown BM, Schwartz RH, Frank E et al. Preoperative evaluation of cervical radiculopathy and myelopathy by surface-coil MR imaging. Am J Roentgenol 1988; 151:1205-1212.

7. Nakstad PH, Hald JK, Bakke SJ et al. MRI in cervical disk herniation. Neuroradiology 1989; 31:382-385.

8. Flanders AE, Schaefer DM, Doan JT et al. Acute cervical spine trauma: Correlation of MRI imaging findings with degree of neurologic deficit. Radiology 1990; 177:25-33.

9. Jahnke RW, Hart BL. Cervical stenosis: spondylosis and herniated disk disease. Rad Clin North Am 1991; 29:777-791.

10. Modic MT, Masaryk TJ, Mulopulos GP et al. Cervical radiculopathy; prospective evaluation with surfacecoil MRI imaging, CT with metrizamide and metrizamide myelography. Radiology 1986; 161:753759.

11. Van de Kleft E, Van Vyve M. Diagnostic imaging algorithm for cervical soft disk herniation. Acta Chir Belg 1995; 95:152-156.

12. El-Khoury GY, Kathol MH, Daniel WW. Imaging of acute injuries of the cervical spine: Value of plain radiography, CT and MR imaging. Am J Roentgenol 1995; 164:43-50.

13. Finelli DA, Hurst GC, Karaman BA et al. Use of magnetization transfer for improved contrast on gradient-echo MRI images of the cervical spine. Radiology 1994; 193:165-171.

14. Melhem ER, Benson ML, Beauchamp NJ et al. Cervical spondylosis. Three-dimensional gradientecho MRI with magnetization transfer. Am J Neuroradiol 1996; 17:705-711.

15. Yousem DM, Atlas SW, Goldberg HI et al. Degenerative narrowing of the cervical spine neural foramina: Evaluation with high-resolution 3-DFT gradient-echo MRI Imaging. Am J Neuroadiol 1991; 12:229-236.

16. Boden SD, McCowin PR, Davis DO et al. Abnormal magnetic resonance scans of the cervical spine in asymptomatic subjects. J Bone Joint Surg (Am) 1990; 72:1178-1184

17. Lehto IJ, Tertti MO, Koomu ME et al. Age-related MRI changes at $0.1 \mathrm{~T}$ in cervical discs in asymptomatic subjects. Neuroradiology 1994; 36:4953.

18. Teresi LM, Lufkin RB, Reicher MA. Asymptomatic degenerative disk disease and spondylosis of the cervical spine: MR imaging. Radiology 1987; 164: 83-88. 